\title{
Control Performance of Thermally Coupled Distillation Sequences with Unidirectional Flows
}

\author{
J. G. Segovia-Hernández ${ }^{1,2}$, S. Hernández ${ }^{2}$, and A. Jiménez ${ }^{1}$ \\ 1 Departamento de Ingeniería Química, Instituto Tecnológico de Celaya \\ E-mail: arturo@iqcelaya.itc.mx \\ 2 Facultad de Química, Universidad de Guanajuato \\ E-mail: [gsegovia, hernasa] @quijote.ugto.mx
}

Summary. The Petlyuk distillation system has been considered with special interest in the field of separation processes because of the high energy savings that it can provide with respect to the operation of sequences based on conventional columns. The original design of the Petlyuk structure, however, shows two interconnections that seem to affect its operational and controllability properties. For one thing, the arrangement is more complex than the common structure of the conventional distillation systems of one feed and two products with no recycle streams. Furthermore, the interconnections show a bidirectional flow of the vapor streams between the two columns of the Petlyuk arrangement, posing a major control challenge for its operation. To overcome this problem, two new structures have been suggested that use unidirectional flows of the vapor or liquid interconnecting streams. The new options have been conceptually developed to improve the controllability properties of the Petlyuk system, but no formal analysis has been conducted on this matter. In this work, a comparative analysis of the control properties of the Petlyuk column and the new arrangements with unidirectional interconnecting flows is presented. Through a singular value decomposition analysis, it is shown that the new schemes provide better theoretical controllability properties than the Petlyuk system. Closed loop tests using proportional-integral controllers were also carried out, and the results showed that, in most of the cases considered, the new arrangements improved the dynamic responses of the Petlyuk column. Such arrangements, therefore, show promising perspectives for its practical consideration.

\section{Introduction}

A chemical process is based on the transformation of raw material into more valuable chemical products, and two of the steps commonly encountered in such processes are the chemical reaction equipment and the section for purification of product species. A common task in the chemical industry, therefore, is the separation of multicomponent mixtures into specified product streams. 

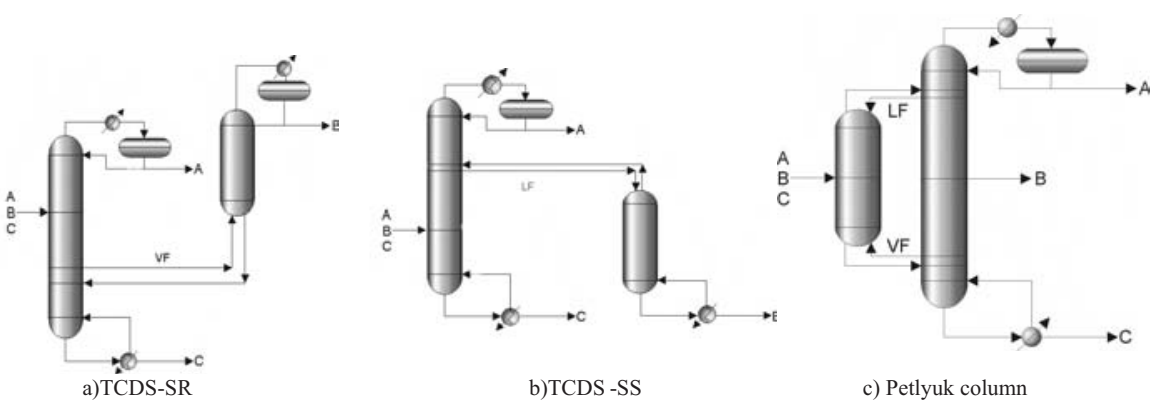

Fig. 1. Three thermally coupled distillation schemes for the separation of ternary mixtures

By far, the dominant choice to carry out such separation tasks is provided by the use of distillation processes; the main disadvantage of the distillation units is that they consume significant amounts of energy, which many times constitute a major portion of the operating costs of the overall process. To improve the energy efficiency of separation processes based on distillation, several strategies have been proposed; one of them calls for the integration of the distillation columns with the rest of the process [24], [33]. The layout of the chemical plant or the energetic characteristics of the streams involved in the process may make such integration limited or not possible, in which case other options can be considered, for example the use of heat pumps or the implementation of thermally coupled arrangements [6], [23], [25]. In particular, the use of columns with thermal coupling has received considerable attention in recent years, with a special development reported for the case of separation problems of ternary mixtures. Thermally coupled distillation systems (TCDS) are obtained through the implementation of interconnecting streams (one in the vapor phase and the other one in the liquid phase) between two columns; each interconnection typically replaces one condenser or one reboiler from one of the columns, thus providing potential savings in capital investment. Furthermore, through a proper selection of the flow values for the interconnecting streams of TCDS, one can obtain significant energy savings with respect to the energy consumption of conventional distillation sequences.

Three thermally coupled schemes have been particularly analyzed. Two of them are fairly similar and make use of a main column and a side column. One can use a side extraction in the vapor phase from the first column and feed it to a side rectifier that purifies the intermediate component. The reboiler of the side column is eliminated by recycling the bottom stream, in the liquid phase, to the first column. The arrangement is known as a thermally coupled distillation system with a side rectifier (TCDS-SR), and its structure is shown in Figure 1a. If the side extraction from the first column is carried out in the liquid phase, a side stripper is used to obtain the intermediate component as a bottom product. In such case, the condenser of the side column is elimi- 
nated if the top vapor stream is sent as a recycle stream to the first column. The resulting structure, a thermally coupled distillation system with a side stripper, TCDS-SS, is shown in Figure 1b. The third option that has been considered with special interest uses two interconnections in an arrangement that consists of a first column (or prefractionator) interconnected through thermal couplings with a main column from which the three components are produced. Through the use of the interconnections, both the condenser and the reboiler of the prefractionator are eliminated. Such option that uses the maximum number of interconnections for the separation of a ternary mixture is referred to as the fully thermally coupled system, or Petlyuk column (Figure 1c).

One of the first studies in which TCDS schemes were taken into account was reported in [35]. The thermally coupled systems with side columns were included as part of a set of eight separation sequences, and they were shown to provide the lowest yearly costs for the separation of ternary mixtures over a wide range of feed compositions. Other theoretical studies followed for the analysis of the structures of Figure 1, most of them under the assumption of minimum reflux conditions. In general, the studies showed that expected savings of minimum internal vapor flows, and therefore of minimum energy requirements, of up to 30 percent could be obtained with respect to the operation of conventional direct and indirect distillation sequences [7], [8], [11], [12], [13]. The savings depend on the amount of the intermediate component in the feed mixture, and are more noticeable for feeds with low or high contents of the intermediate component. More recent works have reported the use of optimization strategies for TCDS to detect designs with minimum energy consumption under un-pinched (finite reflux) conditions [4], [16], [18], [26], [38]. When the performance of the three integrated schemes has been compared, it has been found that in general the Petlyuk system offers better energy savings than the systems with side columns. The energy savings of TCDS can be explained in terms of the internal composition profiles of the intermediate component. The implicit objective of a proper design for a thermally coupled system is to reduce or eliminate the remixing of the intermediate component of the ternary mixture that is typically observed during the operation of a conventional sequence [19], [36]. Thus, if the resulting design of a TCDS scheme is such that the extraction of the side stream is performed at the tray with the maximum internal composition of the intermediate component, then the external energy required for the separation will reach a minimum value for such scheme.

Despite the well-established potential of TCDS in terms of their energy savings, these structures had not been considered for industrial implementation until recent times [22], largely because of the lack of operational experience and to the expectations that they might be rather difficult to control [9]. One may notice the more complex structure of each of the sequences shown in Figure 1, which in general pose higher control challenges than the case of the conventional sequences, in which no recycle streams are used. 
Typically, the control task in distillation processes consists of the production of the main products meeting specified purities. In a conventional distillation column, with one feed and two products, the control of the top product is generally implemented through the adjustment of the reflux flowrate, and the control of the bottom product is tied to the reboiler heat duty. These simple control loops have been used extensively in practice. The TCDS structures, on the other hand, introduce new interconnections for which no operational experience has been gained. Also, a product does not necessarily appear as a top or as a bottom stream. New control loops must be tried, for which no sufficient industrial experience is available. Therefore, a special effort is needed to gain a proper understanding on the control behavior of the more complex TCDS configurations. Some initial works dealing with some of the control aspects of TCDS have recently been reported [1], [17], [20], [29], [30], [32], [37], and in general they have shown that some of these integrated options are controllable, so that the predicted savings in energy would probably not be obtained at the expense of operational and control problems. Further development in this area is still needed.

Another line of research has been observed in recent times, in which efforts to generate alternate structures to the systems of Figure 1, but with more promising expectations as far as their control perspectives, have been suggested. Some newer configurations for TCDS that appear to have some operational advantages over the expected dynamic properties of the designs of Figure 1 have been reported [2], [3], [5], [10], [27], [28]. In particular, the Petlyuk column is the most energy-efficient option, but its structure poses two interesting features. One, it has the highest number of interconnections, and two, the streams in the vapor phase (and therefore also in the liquid phase) flow in the two directions, back and forth between the columns. These aspects affect not only the design but also the control properties of the Petlyuk system. Alternative options can be developed through the correction of each of these two items. Thus, through a reduction on the number of interconnections, or through the use of unidirectional interconnecting flows, one can conceptually generate simpler schemes, in principle easier to control. Six alternative schemes to the Petlyuk column that arise from such modifications have been recently analyzed and reported to show similar energy savings as the original arrangement [21]. These results open a wide interest for the analysis of the dynamic properties of such new schemes; if the options with similar energy efficiencies are proved to provide better controllability properties than the Petlyuk system, then their potential for industrial implementation would be clearly enhanced.

In this work a set of two modifications of the Petlyuk system that use unidirectional flows of both interconnecting streams are considered to analyze their control behavior. The aim of the study is to show if such design modifications may lead to an improvement on the control properties of the original Petlyuk arrangement. 


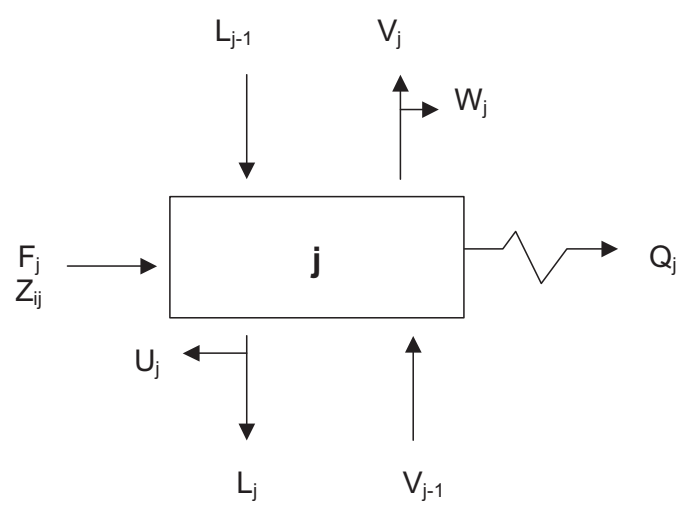

Fig. 2. A generic equilibrium stage for the model of the Petlyuk system

\section{Design and Model of the Petlyuk Column}

A model was first developed for the Petlyuk system. Consider a generic equilibrium stage as shown in Figure 2. The model for the distillation system is based on the following set of equation (with all symbols defined in the Nomenclature section).

Total mass balance:

$$
\frac{d M_{j}}{d t}=L_{j-1}+V_{j+1}+F_{j}^{L}+F_{j}^{V}-\left(L_{j}+U_{j}\right)-\left(V_{j}+W_{j}\right)
$$

Component mass balance:

$$
\begin{aligned}
\frac{d\left(M_{j} X_{i, j}\right)}{d t} & =L_{j-1} X_{i, j-1}+V_{j+1} Y_{i, j+1}+F_{j}^{L} Z_{i, j}^{L}+F_{j}^{V} Z_{i, j}^{V} \\
& -\left(L_{j}+U_{j}\right) X_{i, j}-\left(V_{j}+W_{j}\right) Y_{i, j}
\end{aligned}
$$

Energy balance:

$$
\begin{aligned}
\frac{d\left(M_{j} \bar{U}_{i, j}\right)}{d t} & =L_{j-1} \bar{h}_{j-1}+V_{j+1} \bar{H}_{j+1}+F_{j}^{L} \bar{h}_{j}^{L}+F_{j}^{V} \bar{H}_{j}^{V} \\
& -\left(L_{j}+U_{j}\right) \bar{h}_{j}-\left(V_{j}+W_{j}\right) \bar{H}_{j}+Q_{j}
\end{aligned}
$$

Equilibrium relationships:

$$
Y_{i, j}=K_{i, j} X_{i, j}
$$

Summation constraints:

$$
\sum_{i=1}^{N C} K_{i, j} X_{i, j}-1.0=0
$$


Stage hydraulics (Francis weir formula):

$$
L_{j}=k_{j} L_{W j}\left(h_{O W}\right)_{j}^{3 / 2}
$$

As written, this set of equations provide the dynamic model for the Petlyuk column. Since a design for the column must first be obtained, the same set of equations but written for steady state conditions provides the basis for such a design problem. In any case, the model shows a coupled structure because of the recycle streams between the two columns such that the full set of equations must be solved simultaneously.

The complete design of the Petlyuk system should provide the tray structure of each column, the tray positions for the interconnecting streams, and the operating conditions (such as pressure and reflux ratios or values of the interconnecting streams) that minimize a given objective function, for instance the total yearly cost. This case yields a complicated optimization problem, which would require a mixed-integer nonlinear programming formulation. To overcome such situation, a sequential solution on the tray structure and operating conditions was implemented. For the tray structure, a base design is obtained from the information provided by a conventional distillation system (design methods for conventional distillation systems are well known) consisting of a prefractionator followed by two binary separation columns. Figure 3 shows how the six tray sections of the conventional sequence are related to the structure of the Petlyuk system; such sections perform similar separation tasks in both arrangements. Therefore, the design of the conventional sequence, readily obtained through the use of shortcut methods, provides the tray distribution for the integrated system. The design depends on the operating pressure, which can be set such that the use of refrigerants in the condenser is avoided. Once the design (tray structure) is obtained, it needs to be validated. Steady state rigorous simulations are then conducted to test the preliminary design. If the design specifications (product compositions) are met with the tray structure obtained with the section analogy procedure, the preliminary design was successful; otherwise, proper arrangements in the tray structure are implemented until the specified product compositions are obtained. To complete the design, the validated structure of the Petlyuk system is finally subjected to an optimization procedure to obtain the operating conditions that minimize the energy consumption required for the separation task. Two degrees of freedom remain at this stage, which are used as search variables for the optimization process. The selected search variables are the interconnecting streams of the Petlyuk column (LF and VF, see Figure 1c). Further details on this design procedure are available in [18]. 

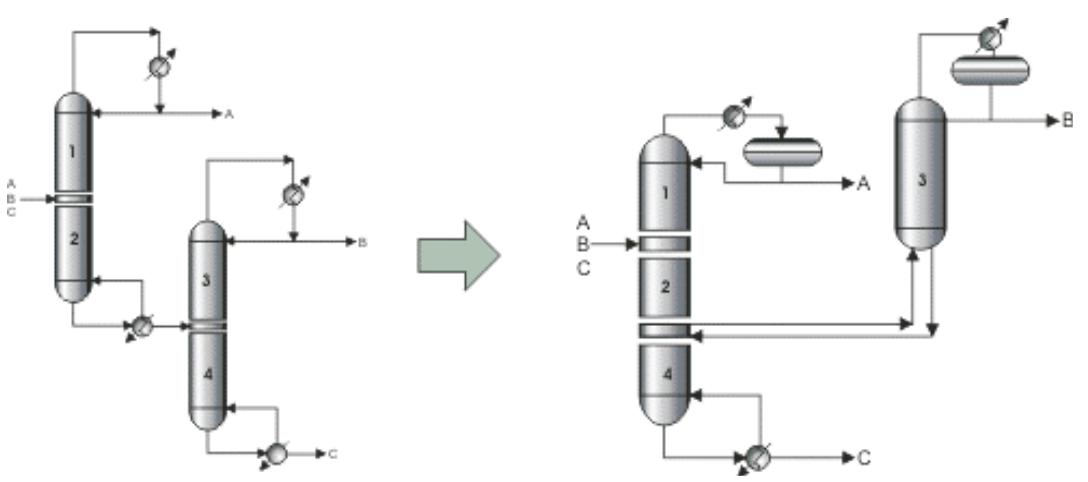

(a)
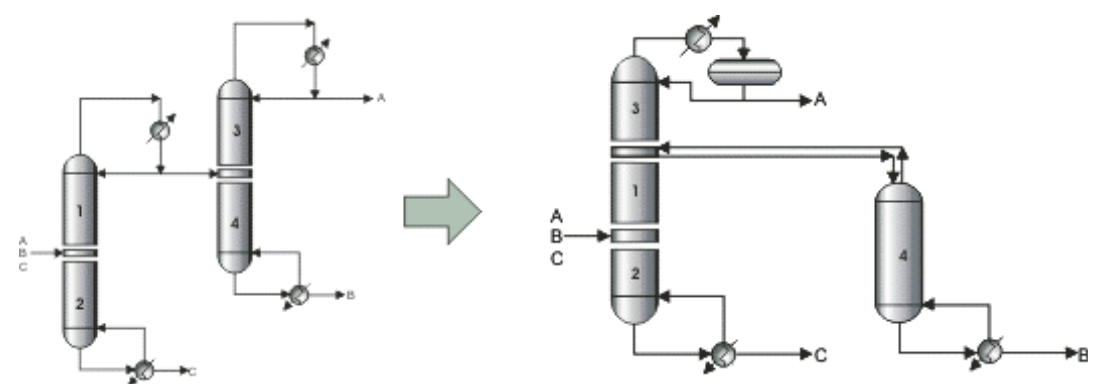

(b)
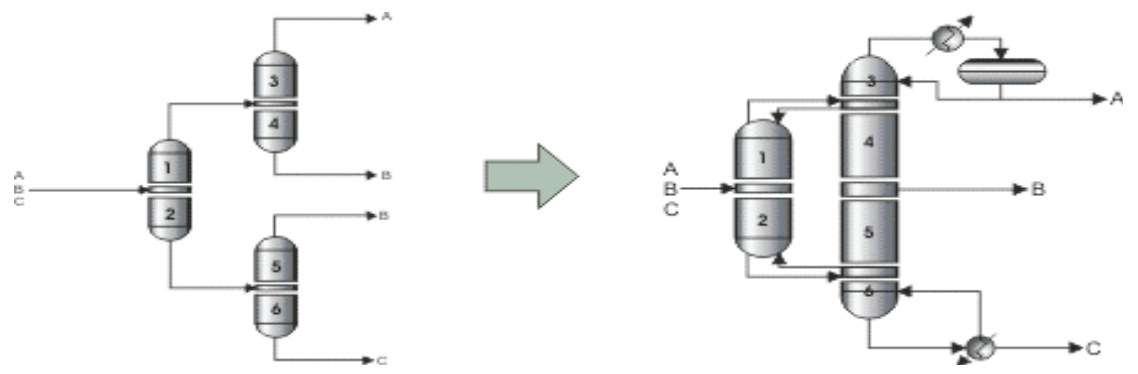

(c)

Fig. 3. Rearrangement of a conventional distillation sequence into the Petlyuk column

3 More Operable Thermally Coupled Distillation Sequences

As mentioned earlier, the Petlyuk column (Figure 1c) poses potential operational problems because of the two directions of the interconnecting vapor 


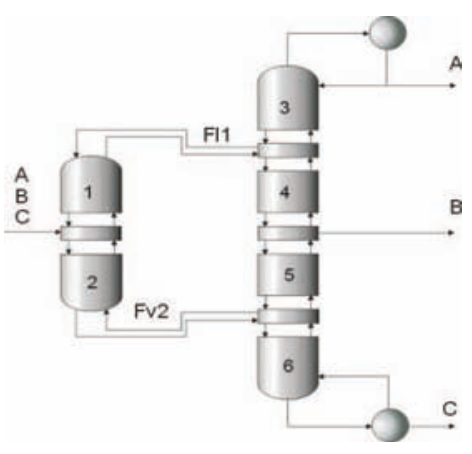

a) Petlyuk Column

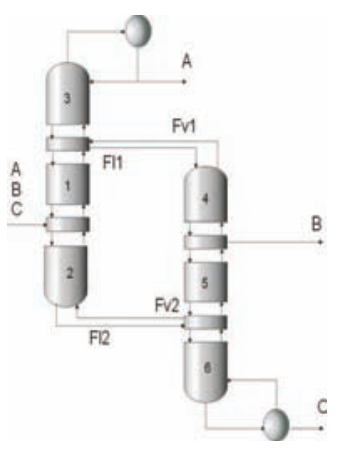

b) Unidirectional arrangement with liquid interconnection

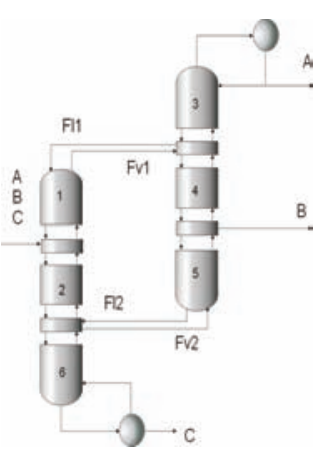

c) Unidirectional arrangement with vapor interconnection

Fig. 4. Alternative schemes to the Petlyuk column

streams. The overhead interconnecting stream of the prefractionator, in the vapor phase, goes from a high pressure point of the prefractionator to a low pressure point of the main column, but the recycle vapor stream from the second column requires a higher pressure of the main column with respect to the prefractionator. This creates an operational conflict since the pressure of neither column can be set higher or lower than the other one. This problem could be avoided, in principle, by implementing unidirectional flows in the interconnecting streams in an alternate arrangement. In [2], [3] have been proposed some of such modifications; two of them are taken in this work for their analysis. Figure $4 \mathrm{~b}$ shows a modified Petlyuk arrangement with unidirectional liquid interconnecting streams from the first column to the second one, while Figure $4 \mathrm{c}$ shows the second option of a modified arrangement with unidirectional vapor interconnecting streams. In the remaining of this work, an analysis on the energy requirements and control properties of the unidirectional alternative schemes of Figures $4 \mathrm{~b}$ and $4 \mathrm{c}$ is conducted, with a comparison shown with respect to those required by the Petlyuk column. 
Table 1. Minimum energy requirements for each arrangement, Btu/h

\begin{tabular}{ccc}
\hline Petlyuk Column & PUL & PUV \\
\hline $1,772,479$ & $1,777,537$ & $1,834,960$ \\
\hline
\end{tabular}

The design of the alternative arrangements is carried out through a section analogy procedure with respect to the design of the Petlyuk system. The system shown in the Figure $4 \mathrm{~b}$ is built by moving tray section 3 from the Petlyuk column (Figure 4a) along with the condenser to the top of the first column. This modification provides an arrangement that is connected at the top of the second column (with a liquid stream) and at the bottom of the first column (with another liquid stream). Both thermal couplings are preserved, but both liquid flows go from the first column to the second column. We identify this configuration as a fully coupled arrangement with liquid flows (PUL). Similarly, one can remove the bottom tray section of the Petlyuk column (section 6 of Figure 4a) with the reboiler and implement them in the bottom part of the first column to produce a fully coupled arrangement with vapor streams (PUV, Figure 4c). The resulting structures with unidirectional interconnecting flows also show two degrees of freedom each, which can be used to find out the operating conditions under which minimum energy consumptions are obtained.

\section{Energy Consumption}

To carry out the analysis, a case study of the separation of a ternary mixture of n-pentane (A), n-hexane (B) and n-heptane (C) with a feed composition low in the intermediate component $(\mathrm{A}, \mathrm{B}, \mathrm{C}$ equal to $0.4,0.2,0.4)$ was considered. The selected composition reflects typical values for which significant energy savings of TCDS have been reported. Design specifications included $98.7 \%$ purity in A, $98 \%$ purity in B and $98.6 \%$ purity in C. A feed flowrate to the sequence of $100 \mathrm{lbmol} / \mathrm{hr}$, available as a saturated liquid, was taken. To avoid the use of refrigerants that would have a high impact on the economics of the separation sequence, the design pressure for each column was chosen such that all condensers could be operated with cooling water. The thermodynamic properties of the mixture were estimated with the Chao-Seader correlation $[15]$.

The first part of the analysis was conducted to detect the designs with minimum energy consumption for the integrated sequences. Once a validated design (tray structure) was obtained, an optimization procedure was carried out on the recycle streams for each of the three coupled sequences to detect the operating conditions under which each design was more energy efficient.

Table 1 shows the values of the minimum energy consumption obtained for the two systems with unidirectional flows and compares them to the one 
obtained for the Petlyuk system. It can be noted that similar energy requirements are obtained for the three options. The energy consumption of the structure with interconnecting vapor streams was slightly higher, but probably because of numerical convergence aspects. The results are consistent with those presented in [21], who conducted a more exhaustive study on the energy consumption of these types of sequences and reported that the new structures have similar energy consumption levels as the Petlyuk scheme. The new sequences are therefore suitable candidates for a complementary analysis on their dynamic properties.

\section{Control Behavior}

The controllability analysis was conducted in two parts. The theoretical control properties of the three schemes were first predicted through the use of the singular value decomposition (SVD) technique, and then closed-loop dynamic simulations were conducted to analyze the control behavior of each system and to compare those results with the theoretical predictions provided by SVD.

\subsection{Singular value decomposition}

The application of the SVD technique provides a measure of the controllability properties of a given dynamic system. More than a quantitative measure, SVD should provide a suitable basis for the comparison of the theoretical control properties among the thermally coupled sequences under consideration. To prepare the information needed for such test, each of the product streams of each of the thermally coupled systems was disturbed with a step change in product composition and the corresponding dynamic responses were obtained. A transfer function matrix relating the product compositions to the intended manipulated variables was then constructed for each case. The transfer function matrix can be subjected to SVD:

$$
G=V \Sigma W^{H}
$$

where $\Sigma=\operatorname{diag}\left(\sigma_{1} \ldots \sigma_{n}\right), \sigma_{1}=$ singular value of $G=\lambda_{i}^{1 / 2}\left(G G^{H}\right) ; V=$ $\left(V_{1}, V_{2}, \ldots\right)$ matrix of left singular vectors, and $W=\left(w_{1}, w_{2}, \ldots\right)$ matrix of right singular vectors. Two parameters of interest are the minimum singular value, $\sigma_{*}$, and the ratio of maximum to minimum singular values, or condition number:

$$
\gamma^{*}=\sigma^{*} / \sigma_{*}
$$

The minimum singular value is a measure of the invertibility of the system and therefore represents a measure of the potential problems of the system under feedback control. The condition number reflects the sensitivity of the system under uncertainties in process parameters and modeling errors. These 
Table 2. Minimum singular value and condition number for each arrangement

\begin{tabular}{ccc}
\hline Sequence & $\sigma_{*}$ & $\gamma^{*}$ \\
\hline Petlyuk Column & 0.0055 & 1260.47 \\
PUL & 0.4383 & 14.33 \\
PUV & 0.0363 & 252.68 \\
\hline
\end{tabular}

parameters provide a qualitative assessment of the dynamic properties of a given design. The objective is to identify the systems with higher minimum singular values and lower condition numbers; those systems are expected to show the best dynamic performance under feedback control. A nice feature of the SVD test is that it is independent of the type of controller to be implemented; the basic idea is that the controllability properties of the system are instead limited or imposed by its inner dynamic structure.

The full application of the SVD method should consider a range of frequencies sufficiently high to give a complete coverage on the behavior of minimum singular values and conditions numbers, as shown in [17], [20] for their controllability analysis of some conventional and nonconventional distillation systems. As a preliminary attempt, however, the SVD analysis was conducted in this work only at zero frequency. Such analysis requires only the steadystate gain matrix, and gives the theoretical controllability properties of each system around its nominal operating point, in this case around the point for which a minimum energy consumption was obtained. This information should be sufficient at this point for the intended comparative analysis on the controllability properties of the three thermally coupled systems.

Table 2 shows the results obtained from the application of the SVD at zero frequency for each sequence. The PUL column has the lowest value of the condition number, which implies that this sequence is better conditioned to the effect of disturbances than the other two integrated systems; it also presents the highest value of $\sigma_{*}$, which means that the sequence is expected to show the lowest control effort. Therefore, from this initial analysis, it is apparent that the PUL scheme will show the best operational properties of the three options under consideration. On the other hand, it is interesting to notice that the Petlyuk column offers the worst theoretical control properties (lowest minimum singular value and highest condition number), which supports the notion that the new arrangements may indeed improve the control properties of the Petlyuk system through the correction of the bidirectionality of the vapor interconnecting streams observed in the original design.

\subsection{Closed-loop simulations}

To supplement the SVD analysis, rigorous dynamic simulations under closed loop operation were carried out. For the closed-loop analysis, several issues must be defined first, such as the control loops for each system, the type of 
process controller to be used, and the values of the controller parameters. Several techniques, such the relative gain array method, can be used to fix the loops for a control system. In the case of distillation columns, however, such loops are fairly well established and used successfully in practice, at least for conventional columns. A well-known structure is based on energy balance considerations, which yields to so-called LV control structure in which the reflux flowrate $L$ and the vapor boilup rate $V$ (affected directly by the heat duty supplied to the reboiler) are used to control the distillate and bottom outputs compositions (see for instance [14]). The control loops for the integrated systems were chosen from extensions of the practical considerations observed for conventional distillation columns. The control objective was to preserve the output streams at their design purity specifications. Two control loops arise naturally from the experience on the operation of conventional columns. For the control of product stream A, that is obtained as an overhead product, the reflux flowrate was used, whereas for the control of product stream $\mathrm{C}$, that is obtained as a bottom product, the reboiler heat duty was chosen. Product stream B is obtained as a side stream for the integrated arrangements, and in the lack of operational experience, its control was simply set through the manipulation of the side stream flow rate. It should be mentioned that such control loops have been used with satisfactory results in previous studies we have conducted on thermally coupled systems [20], [29], [30], [31]. The choice of the type of controller was based on the ample use that the ProportionalIntegral (PI) mode has for distillation systems in industrial practice. Also, since this is the first reported analysis on the controllability properties of the alternative schemes of figures $4 \mathrm{~b}$ and $4 \mathrm{c}$, the choice may also provide a basis upon which the use of more elaborated control laws can be compared. As for the selection of the parameters of the PI controllers, care was taken to provide a common method for each of the sequences under comparison. A tuning procedure that involved the minimization of the integral of the absolute value (IAE) for each loop of each scheme was used [34]. Therefore, for each loop, an initial value of the proportional gain was set; a search over the values of the integral reset time was conducted until a local optimum value of the IAE was obtained. The process was repeated for other values of the proportional gain. The selected set of controller parameters was then the one that provided a global minimum value of the IAE. Although the tuning procedure is fairly elaborated, the control analysis is conducted based on a common tuning method for the controller parameters, Table 3 shows the parameters obtained for the control of each product stream.

The simulations involve the solution of the rigorous tray-by-tray model of each sequence, given by equations 1 to 6 , together with the standard equations for the PI controllers for each control loop (with the parameters obtained through the minimization of the IAE criterion). The objective of the simulations is to find out how the dynamic behavior of the systems compare under feedback control mode. To carry out the closed-loop analysis, two types of cases were considered: i) servo control, in which a step change was induced 
Table 3. Parameters of the PI controllers for each control loop

\begin{tabular}{ccc}
\hline Sequence & $\tau_{l}($ min $)$ & $K_{c}(\% / \%)$ \\
\hline Petlyuk Column & $\mathrm{A}=30$ & $\mathrm{~A}=20$ \\
& $\mathrm{~B}=5$ & $\mathrm{~B}=130$ \\
& $\mathrm{C}=10$ & $\mathrm{C}=20$ \\
\hline PUL & $\mathrm{A}=60$ & $\mathrm{~A}=80$ \\
& $\mathrm{~B}=150$ & $\mathrm{~B}=140$ \\
& $\mathrm{C}=10$ & $\mathrm{C}=100$ \\
\hline PUV & $\mathrm{A}=70$ & $\mathrm{~A}=20$ \\
& $\mathrm{~B}=40$ & $\mathrm{~B}=40$ \\
& $\mathrm{C}=30$ & $\mathrm{C}=180$ \\
\hline
\end{tabular}

in the set point for each product composition under SISO feedback control, and ii) regulatory control, in which a feed disturbance with a $5 \%$ change in the composition of one component (with a proportional adjustment in the composition of the other components to keep the same total feed flowrate) was implemented.

\section{Dynamic behavior of the heavy component (set point change)}

When a set point change from 0.986 to 0.99 for the heavy component (C) was considered, the responses shown in Figure 5 were obtained. The three options show good dynamic responses, with relatively low values of settling times, lower than 0.1 hours. The systems with unidirectional flows show some overshooting, while the Petlyuk system offers a more sluggish response.

In addition to the visual observations of the dynamic responses, a quantitative measure is needed to provide a better comparison. With such an objective, IAE values were evaluated for each closed-loop response. The PUL option shows the lowest IAE value of $5.607 \times 10^{-5}$, while the value for the Petlyuk column turns out to be $2.35 \times 10^{-4}$. Therefore, the results of the test indicate that, for the SISO control of the heaviest component of the ternary mixture, the PUL option provides the best dynamic behavior and improves the performance of the Petlyuk column. Such result is consistent with the prediction provided by the SVD analysis.

\section{Dynamic behavior of the light component (set point change)}

The results of the dynamic test for a positive change in the set point of the light component (A) are displayed in Figure 6. For a change in the set point from 0.987 to 0.991 , the three systems are shown to be controllable and reach the new value of product composition, although the PUL scheme shows a quicker adjustment. IAE values were also calculated for each response; the two best 

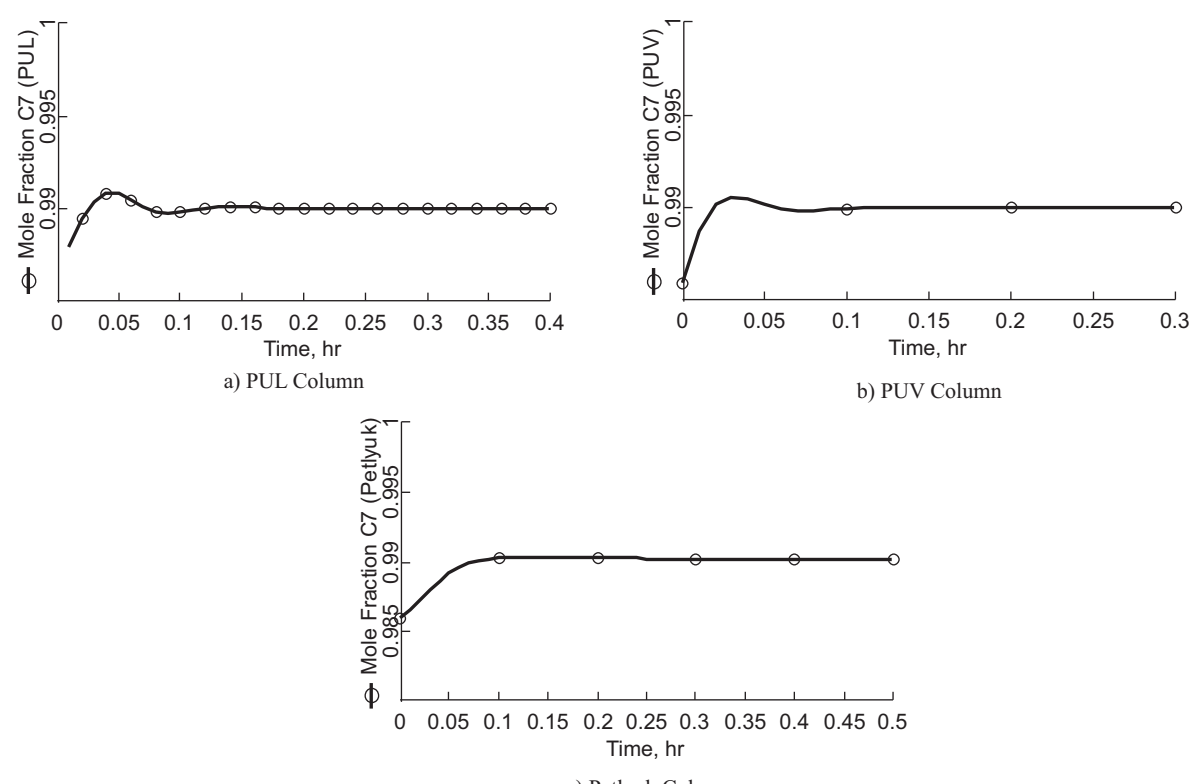

Fig. 5. Closed loop responses for a set point change in the composition of the heavy component

IAE values correspond to the new arrangements: $4.20 \times 10^{-5}$ for the PUL system, and $6.10 \times 10^{-5}$ for the PUV system. The IAE value for the Petlyuk column was $2.35 \times 10^{-4}$, which again shows a case in which the dynamic properties of the Petlyuk system are improved through the modifications that provide unidirectional flows of the interconnecting streams.

\section{Dynamic behavior of the intermediate component (set point change)}

Figure 7 shows the dynamic responses obtained when the set point for the intermediate component was changed from 0.98 to 0.984 . One may notice the better response provided by the Petlyuk column in this case, which is faster than the other two systems and without oscillations. When the IAE values were calculated, a remarkable difference in favor of the Petlyuk system was observed: $2.87 \times 10^{-4}$ for the Petlyuk column, compared to 0.0011 for the PUL system and 0.0017 for the PUV system. The results from this test may seem unexpected, since the new arrangements have been proposed to improve the operation capabilities of the Petlyuk column. The SISO control of the intermediate component, interestingly, seems to conflict with that of the other two components in terms of the preferred choice from dynamic considerations. 


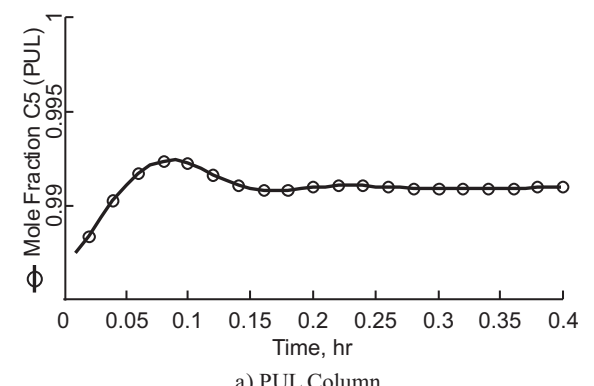

a) PUL Column

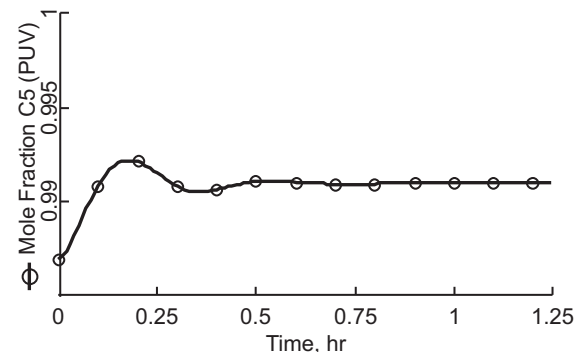

b) PUV Column

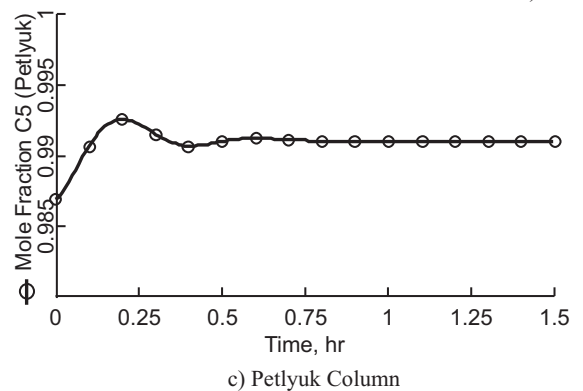

Fig. 6. Closed loop responses for a set point change in the composition of the light component

\section{Feed disturbance analysis}

The last set of tests conducted in this work had to do with the implementation of feed disturbances. The regulatory control tests consisted of a change in the feed composition with the same nominal feed flowrate. Under such scenario, it is clear that the feed composition of all components must change, but the component with the highest change (of 5 percent) was used to identify the tests. Figure 8 shows the results obtained when a feed disturbance in the composition of component A was implemented. The PI controllers successfully rejected the feed disturbance for each of the separation systems, although the settling times are higher than those obtained for the servo tests. It can be observed that, consistent with the SVD results, the best response of the three alternatives is provided by the PUL system, which also implies a lower control effort. When the systems were compared according to their IAE values, the Petlyuk column showed the highest value of 0.0018 .

The results for this test and for the responses to disturbances in feed compositions of $\mathrm{B}$ and $\mathrm{C}$ are summarized in Table 4 . A consistent trend with the servo tests was observed, in the sense that one option provides the best common choice for the control of the system under feed disturbances on the extreme components of the mixture, but a different arrangement yields a superior dynamic performance for the control task under a feed disturbance on the intermediate component. From Table 4, the IAE values indicate that 


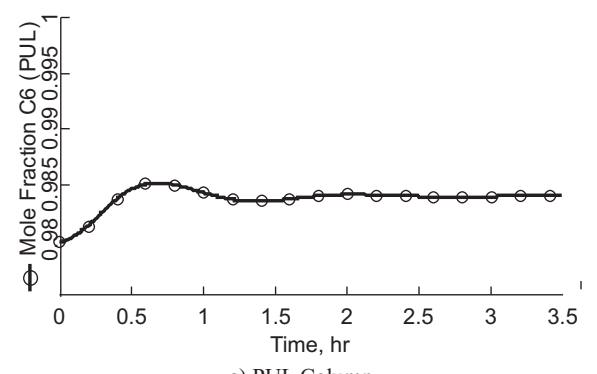

a) PUL Column

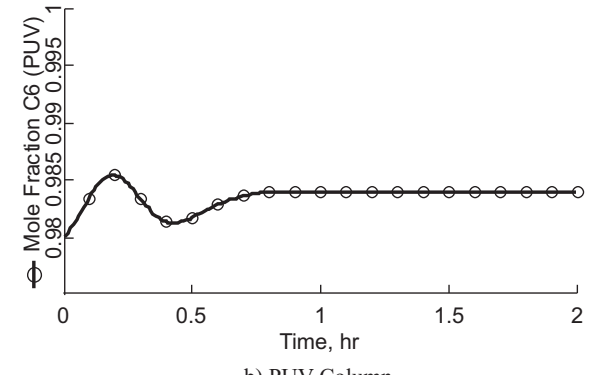

b) PUV Column

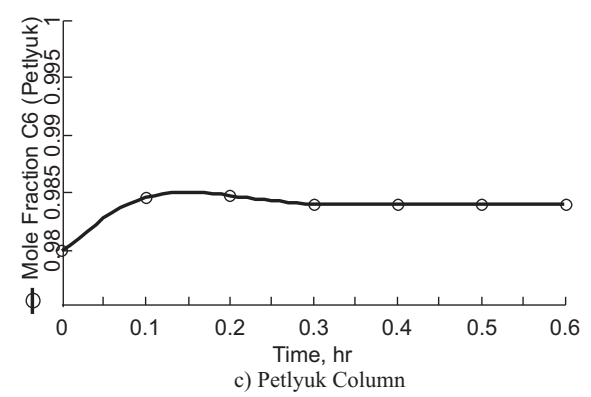

Fig. 7. Closed loop responses for a set point change in the composition of the intermediate component

the PUL system shows the best behavior for feed disturbances in the light and heavy component. However, the PUL arrangement shows the worst response when the feed disturbance in the intermediate component was considered, in which case both the Petlyuk and the PUV systems show fairly similar rejection capabilities.

Table 4. IAE values for the feed disturbance test

\begin{tabular}{cccc}
\hline Sequence & A & B & C \\
\hline Petlyuk Column & 0.0018 & $1.66 \times 10^{-4}$ & $5.83 \times 10^{-4}$ \\
PUL & $3.63 \times 10^{-4}$ & 0.0059 & $9.60 \times 10^{-5}$ \\
PUV & 0.0011 & $1.50 \times 10^{-4}$ & $6.20 \times 10^{-4}$ \\
\hline
\end{tabular}

\section{Conclusions}

To improve the low energy efficiency observed during the operation of conventional distillation sequences, some integrated arrangements have been sug- 

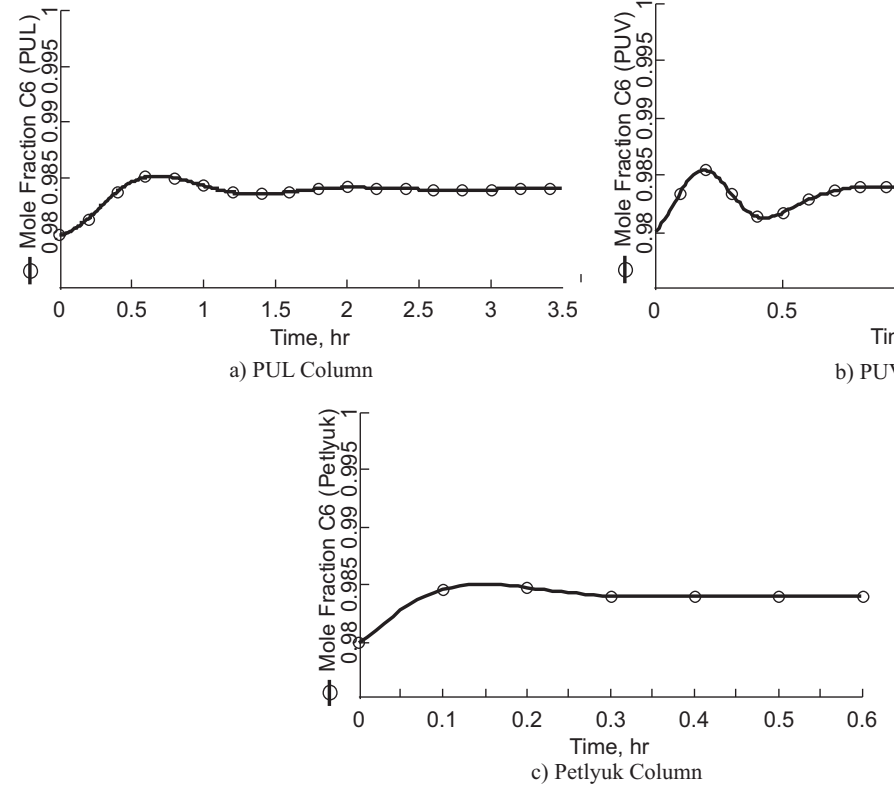

Fig. 8. Closed loop responses for feed disturbance in the composition of the light component

gested. Distillation sequences with thermal coupling have been shown to provide promising options because of their potential energy savings. Among these options, the Petlyuk configuration stands as the most efficient arrangement in terms of its energy requirements. Originally developed for the separation of ternary mixtures, the Petyuk system shows two thermal couplings whose structure seems to conflict in terms of its operational perspectives. The thermal couplings show a bidirectional flow of the vapor interconnecting streams, which implies that it is not possible to design one of the columns with a pressure uniformly higher than the other one. Among the possible revisions that have been suggested to improve the operational perspectives of the Petlyuk system, two modifications that keep the same number of thermal couplings but use a unidirectional flow of the interconnecting (liquid or vapor) streams from the first column to the second one have been suggested. An analysis on the dynamic properties of the two new schemes with unidirectional flows of the interconnecting streams has been conducted in this work and compared to the behavior of the original Petlyuk arrangement. A simplified analysis via singular value decomposition at zero frequency indicates that the new arrangements may improve the controllability properties around the nominal operating point with respect to the Petlyuk system. A set of rigorous dynamic simulations under closed loop operation with PI controllers was also conducted. The closed loop simulations were based on a SISO control strategy, and the results showed an interesting trend. The control behavior of the Pet- 
lyuk column was improved by the new options when the control tasks called for the control of the extreme components ( $\mathrm{A}$ or $\mathrm{C}$ ) of the ternary mixture. However, when the control of the intermediate component was considered, the Petlyuk column provided a better dynamic performance. Overall, the results here obtained show that the new arrangements with unidirectional interconnections provide interesting choices for further consideration. For one thing, they seem to preserve the energy efficiency of the original Petlyuk system; for the other, they were shown to offer proper controllability properties. In particular, the structure with unidirectional liquid interconnections provided the best theoretical control properties, and yielded the best overall dynamic behavior under the operation scenarios considered in this work.

\title{
List of Abbreviations and Symbols
}

\author{
Related to the process: \\ $F \quad$ feed flow-rate (lb-mol/hr) \\ $\bar{H} \quad$ enthalpy of vapor $(\mathrm{Btu} / \mathrm{hr})$ \\ $\bar{h} \quad$ enthalpy of liquid $(\mathrm{Btu} / \mathrm{hr})$ \\ $h_{O W} \quad$ liquid height on weir (in) \\ $K \quad$ equilibrium constant \\ $L \quad$ liquid flow-rate (lb-mol/hr) \\ $L_{W} \quad$ weir length (in) \\ $M \quad$ moles of liquid retained (lb-mol) \\ $Q \quad$ heat added or extracted (Btu/hr) \\ $t \quad$ time $(\mathrm{hr})$ \\ $U \quad$ liquid side stream (lb-mol/hr) \\ $\bar{U} \quad$ internal energy of liquid retained (Btu/hr) \\ $u \quad$ manipulated input \\ $V \quad$ vapor flow-rate (lb-mol/hr) \\ $W \quad$ vapor side stream $(\mathrm{lb}-\mathrm{mol} / \mathrm{hr})$ \\ $X \quad$ mole fraction in liquid phase \\ $Y \quad$ mole fraction in vapor phase
}

Superscripts:

L liquid

vapor

Subscripts:

$i$ component

$j \quad$ stage 


\section{References}

[1] M.I. Abdul-Mutalib and R. Smith. Operation and control of dividing wall distillation columns. Part I: Degrees of freedom and dynamic simulation. Trans. IchemE, 76(Part A):308-318, 1998.

[2] R. Agrawal and Z. Fidkowski. More operable arrangements of fully thermally coupled distillation columns. A.I.Ch.E. Journal, 44(11):2565-2568, 1998.

[3] R. Agrawal and Z. Fidkowski. New thermally coupled schemes for ternary distillation. A.I.Ch.E. Journal, 45(3):485-496, 1999.

[4] K.A. Amminudin, R. Smith, D.Y.C. Thong, and G.P. Towler. Design and optimization of fully thermally coupled distillation columns. Part I: Preliminary design and optimization methodology. Trans. IchemE, 79(Part A):701-715, 2001.

[5] O. Annakou, A. Meszaros, Z. Fonyo, and P. Mizsey. Operability investigation of energy integrated distillation schemes. Hung. J. Ind. Chem., 24:155-160, 1996.

[6] O. Annakou and P. Mizsey. Rigorous investigation of heat pump assisted distillation. Heat Recovery Syst. CHP, 15(3):241-247, 1995.

[7] O. Annakou and P. Mizsey. Rigorous comparative study of energy integrated distillation schemes. Ind. Eng. Chem. Res., 35:1877-1885, 1996.

[8] J. Cerda and A.W. Westerberg. Shortcut methods for complex distillation columns. I. Minimum reflux. Ind. Eng. Chem. Process Des. Dev., 20:546$557,1981$.

[9] G. Dünnebier and C. Pantelides. Optimal design of thermally coupled distillation columns. Ind. Eng. Chem. Res., 38:162-176, 1999.

[10] M. Emtir, P. Mizsey, E. Rev, and Z. Fonyo. Economic and controllability investigation and comparison of energy - integrated distillation schemes. Chem. Biochem. Eng. Q., 17(1):31-40, 2003.

[11] Z. Fidkowski and L. Krolikowski. Energy requirements of nonconventional distillation systems. A.I.Ch.E. Journal, 36(8):1275-1278, 1991.

[12] A. Finn. Consider thermally coupled distillation. Chem. Eng. Prog., 10:41-45, 1993.

[13] K. Glinos and M.F. Malone. Optimality regions for complex column alternatives in distillation systems. Chem. Eng. Res. Des., 66:229-240, 1988.

[14] K.E. Häggblom and K.V. Waller. Practical Distillation Control, chapter Control Structures, Consistency, and Transformations. Van Nostrand Reinhold, New York, 1992.

[15] E.J. Henley and J.D. Seader. Equilibrium-Stage Separation Operations in Chemical Engineering. Wiley, New York, 1981.

[16] S. Hernández and A. Jiménez. Design of optimal thermally - coupled distillation systems using a dynamic model. Trans. IchemE, 74(Part A):357-362, 1996. 
[17] S. Hernández and A. Jiménez. Controllability analysis of thermally coupled distillation systems. Ind. Eng. Chem. Res., 38(10):3957-3963, 1999.

[18] S. Hernández and A. Jiménez. Design of energy - efficient petlyuk systems. 23(8):1005-1010, 1999.

[19] S. Hernández, S. Pereira-Pech, and V. Rico-Ramírez. Energy efficiency of an indirect thermally coupled distillation sequence. Can. J. Chem. Eng., 81(5):1087-1091, 2003.

[20] A. Jiménez, S. Hernández, F.A. Montoy, and M. Zavala-García. Analysis of control properties of conventional and nonconventional distillation sequences. Ind. Eng. Chem. Res., 40(17):3757-3761, 2001.

[21] A. Jiménez, N. Ramírez, A. Castro, and S. Hernández. Design and energy performance of alternative schemes to the petlyuk distillation system. Trans. IchemE, 81(Part A):518-524, 2003.

[22] G. Kaibel and H. Schoenmakers. Process synthesis and design in industrial practice. In J. Grievink and J.V. Schijndel, editors, European Symposium on Computer Aided Process Engineering-12, pages 9-22. Elsevier, Holland, 2002.

[23] I. Meszaros and Z. Fonyo. A new bounding strategy for synthesising distillation schemes with energy integration. Comput. Chem. Eng., 10:545$560,1986$.

[24] P. Mizsey and Z. Fonyo. Toward a more realistic process synthesis - The combined approach. Comput. Chem. Eng., 14(11):1303-1310, 1990.

[25] P. Mizsey and Z. Fonyo. Energy integrated distillation system design enhanced by heat pumping distillation and absorption. Inst. Chem. Eng., pages B69-B75, 1992.

[26] K. Muralikrishna, K.P. Madhavan, and S.S. Shah. Development of dividing wall distillation column design space for a specified separation. Trans. IchemE, 80(Part A):155-165, 2002.

[27] B.G. Rong and A. Kraslawski. Optimal design of distillation flowsheets with a lower number of thermal couplings for multicomponent separations. Ind. Eng. Chem. Res., 41:5716-5726, 2002.

[28] B.G. Rong and A. Kraslawski. Partially thermally coupled distillation systems for multicomponent separations. A.I.Ch.E. Journal, 49:13401347, 2003.

[29] J.G. Segovia-Hernández, S. Hernández, and A. Jiménez. Análisis dinámico de secuencias de destilación térmicamente acopladas (in spanish). Información Tecnológica, 13:103-108, 2002.

[30] J.G. Segovia-Hernández, S. Hernández, and A. Jiménez. Control behaviour of thermally coupled distillation sequences. Trans. IchemE, 80(Part A):783-789, 2002.

[31] J.G. Segovia-Hernández, S. Hernández, V. Rico-Ramírez, and A. Jiménez. A comparison of the feedback control behavior between thermally coupled and conventional distillation schemes. Comput. Chem. Eng., 28:811-819, 2004. 
[32] M. Serra, A. Espuña, and L. Puigjaner. Control and optimization of the divided wall column. Chem. Eng. Process., 38:549-562, 1999.

[33] R. Smith and B. Linnhoff. The design of separators in the context of overall process. Chem. Eng. Res. Des., 66:195-210, 1988.

[34] G. Stephanopoulos. Chemical Process Control: An introduction to theory and practice. Prentice Hall, New Jersey, 1984.

[35] D.W. Tedder and D.F. Rudd. Parametric studies in industrial distillation: Part I. Design comparisons. A.I.Ch.E. Journal, 24:303-315, 1978.

[36] C. Triantafyllou and R. Smith. The design and optimization of fully thermally coupled distillation columns. Trans. Inst. Chem. Eng., 70(Part A):118-132, 1992.

[37] E. Wolff and S. Skogestad. Operation of integrated three - products (petlyuk) distillation columns. Ind. Eng. Chem. Res., 34(6):2094-2103, 1995.

[38] H. Yeomans and I. Grossmann. Optimal design of complex distillation columns using rigorous tray-by-tray disjunctive programming models. Ind. Eng. Chem. Res., 39(11):4326-4335, 2000. 